\title{
Self-report depressive symptoms do not directly predict suicidality in nonclinical individuals: Contributions toward a more psychosocial approach to suicide risk
}

\author{
Rui C. Campos ${ }^{\mathrm{a}}$, Ronald R. Holden ${ }^{\mathrm{b}}$, Patrícia Laranjeirac, Talia Troister ${ }^{\mathrm{b}}$, Ana Rita Oliveirac, Fátima Costac, \\ Marta $\mathrm{Abreu}^{\mathrm{c}}$, and Natália Frescac \\ aDepartamento de Psicologia, Escola de Ciências Sociais e Centro de Investigação em Educação e Psicologia, University of Évora, Évora, \\ Portugal; 'Department of Psychology, Queen's University, Kingston, Ontario, Canada; 'Departamento de Psicologia, Escola de Ciências Sociais, \\ University of Évora, Évora, Portugal
}

\begin{abstract}
Although suicidality is associated with mental illness in general and depression in particular, many depressed individuals do not attempt suicide and some individuals who attempt to or do die by suicide do not present depressive symptoms. This article aims to contribute to a more psychosocial approach to understanding suicide risk in nonclinical populations. In advocating a psychosocial perspective rather than a depression-focused approach, this article presents four diverse studies that demonstrate sampling and measurement invariance in findings across different populations and specific measures. Study 1 tests the mediation effects of 2 interpersonal variables, thwarted belongingness and perceived burdensomeness, in the association between depressive symptoms and recent suicidality. Studies 2 and 3 evaluate the contribution of hopelessness and psychache, beyond depressive symptoms, to suicidality. Study 4 tests the contribution of life events behind depressive symptoms, and other relevant sociodemographic and clinical variables, to the estimation of "future suicidality." Overall, results demonstrate that depressive symptoms do not directly predict suicidality in nonclinical individuals, but that other psychosocial variables mediate the association between depressive symptoms and suicidality or predict suicidality when statistically controlling for depressive symptoms. The article contributes to understanding some of the nonpsychopathological factors that potentially link depressive symptoms to suicide risk and that might themselves contribute to suicidality, even when controlling for depressive symptoms.
\end{abstract}

Suicide is a major public health issue in both clinical and nonclinical populations. The World Health Organization (2012) estimates that each year almost one million people die by suicide worldwide. Nonfatal suicidal behaviors, such as suicide attempts and selfdirected aggression, are estimated to occur 10 to 20 times more often than completed suicide (World Health Organization, 2012).

The presence of a psychiatric disorder in individuals who have died by suicide or attempted to kill themselves is very frequent (e.g., Cavanagh, Carson, Sharpe, \& Lawrie, 2003). For example, in a longitudinal study, Borges, Angst, Nock, Ruscio, and Kessler (2008) demonstrated that mental illness predicted suicidal behaviors, including ideation, plans, and attempts. The presence of a depressive disorder, in particular, is even more strongly related to suicidality; suicidality is, in fact, a symptom of depressive disorders. Depression and bipolar depression are the most typical psychiatric disorders present among suicide completers (e.g., Khan et al., 2008) and, among depressed suicide completers, $48 \%$ had previously attempted suicide (Isometsa et al., 1994).

But this clinical and epidemiological evidence, although providing important information for risk identification and therapeutic intervention, raises at least three compelling questions: Why do some mentally ill or clinically depressed individuals not attempt to die by suicide or even present suicide ideation? When a psychiatric disorder exists, why does it augment the probability for suicidality (i.e., what is the nature of this link)? And third, and perhaps even more important, why do some individuals, despite not having a psychiatric disorder, display suicide ideation or behaviors? This last question leads to an important issue in

CONTACT Rui C. Campos rcampos@uevora.pt Đ Departamento de Psicologia, Escola de Ciências Sociais e Centro de Investigação em Educação e Psicologia, Universidade de Évora, Apartado 94, Évora, 7002-554, Portugal.

Color versions of one or more of the figures in the article can be found online at www.tandfonline.com/udst.

(c) 2016 Taylor \& Francis 